\title{
Recent exposure affects artifact naming
}

\author{
STEVEN A. SLOMAN and MARIANNE C. HARRISON \\ Brown University, Providence, Rhode Island \\ and \\ BARBARA C. MALT \\ Lehigh University, Bethlehem, Pennsylvania
}

\begin{abstract}
Deciding how to label an object depends both on beliefs about the culturally appropriate name and on memory. A label should be consistent with a language community's norms, but those norms can be used only if they can be retrieved. Two experiments are reported in which we tested the hypothesis that immediate prior exposure to familiar objects and their names affects how an ambiguous target object is named. Exposure to a typical instance of one name category was pitted against exposure to one or two instances from a contrasting category. When the contrast set consisted of a neighbor of the target, naming was usually consistent with the contrast category. This effect was reduced when a typical instance of the contrast category was also exposed. In Experiment 2, the exposure set was varied to include conditions in which either the neighbor or a prototypical instance was paired with an instance dissimilar to the target. The results suggest that all recently exposed objects affect name choice in proportion to their similarity to the target.
\end{abstract}

Say that one wants to refer to an object. Several factors will contribute to what one calls it. First, the constraints of one's language come into play. Languages make some labels appropriate and others not. A mechanic who called the rubber blades that sweep across the windshield of a car a radio would be uncooperative, because in English such things are called wipers. We call such constraints on naming the conventionalmeaning of a word, because they are housed in the conventions of a linguistic community. Second, name choice is affected by a speaker's goals and other contextually determined pragmatic considerations (e.g., Brown, 1958; Grice, 1975). For example, participants in a conversation might choose specific terms as part of a pact to adopt a specific conceptualization of an object (Brennan \& Clark, 1996). Third, one's answer will depend on memory. One must retrieve a term in order to use it, along with some idea of the set of objects that the term normally applies to. The purpose of this paper is to examine how pervasive the effect of memory for a reference set is and how memory is used for naming. Specifically, we investigated how naming an ambiguous object would be affected by recent exposure to common and familiar objects that vary in their similarity to the target object and in their typicality.

A variety of studies have already documented the effects of prior exposure to specific instances on related tasks. Changing the range of recently observed instances by

This work was supported by NIMH Grant MH51271 to B.C.M. and S.A.S. We thank Brian Ross, Evan Heit, and Bradley Franks for their input. Correspondence concerning this article should be addressed to S.A. Sloman, Cognitive and Linguistic Sciences, Brown University, Box 1978, Providence, RI 02912 (e-mail: stevensloman@brown.edu). presenting more or less extreme examples on a dimension can cause various sorts of judgments to assimilate to the presented examples (e.g., Ward \& Lockhead, 1970, which used judgments of loudness), to contrast with the presented examples (e.g., Wedell \& Pettibone, 1999, which used preference judgments for schematic faces), or both (Bless, Igou, Schwarz, \& Wänke, 2000, which used political judgments). The frequency of exposure to specific instances and the similarity of specific instances to members of a contrast category affect learning and typicality judgments in artificial categories (e.g., Medin \& Schaffer, 1978; Nosofsky, 1988). Comparison with specific instances is a common strategy in category learning and affects judgments of feature frequency and feature correlations (Ross, Perkins, \& Tenpenny, 1990; Spalding \& Ross, 1994). Heit (1992) and Livingston, Andrews, and Dwyer (2001) have shown that categorization decisions can be based on chains of specific examples (for related evidence, see Malt, Sloman, Gennari, Shi, \& Wang, 1999). Similarity to previously presented exemplars affects how features are interpreted (Spalding \& Ross, 2000). Prior exposure influences the comparison processes involved in induction (Medin \& Ross, 1989).

Despite all this research, few studies have examined the effect of prior exposure on classification in well-learned, familiar categories. An exception to this is a study by Brooks, Norman, and Allen (1991), who found that prior exposure to specific examples of dermatological lesions influenced subsequent diagnostic classifications of lesions by both medical residents and experienced physicians. The effect depended on memory for specific exemplars: The more similar the lesion was to lesions shown earlier, the greater was the effect on classification. 
The present experiments test whether this type of result extends to nouns like chair, pen, and broom that are used to label common artifacts. Prior research showing dissociations between naming and nonlinguistic categorization tasks (Gennari, Sloman, Malt, \& Fitch, 2002; Malt et al., 1999; Sloman \& Ahn, 1999) implies that results shown with one kind of categorization task do not necessarily extend to other, especially linguistic, categorization tasks. In particular, naming serves a communicative function and so makes demands distinct from those of other categorization tasks. So the extent and nature of any effect of prior exposure on the naming of common objects remains unknown.

If conventional meaning alone determines how people name objects, recent prior exposure to specific exemplars should not measurably affect name choice. The conventional meaning of a word has been construed in many ways: as an essence (e.g., Putnam, 1975), as a belief about intended category membership (Bloom, 1996), as a shared cognitive schema grounded in bodily experience (Lakoff, 1987), as a pointer to a substance of a certain type (Millikan, 1998), or as a prototype (Taylor, 1995). Whatever one's view of conventional meaning, it cannot explain any influence of an individual's recent exposure to exemplars on a word's subsequent use. Merely hearing a particular boat called a skiff may make one more likely to call a similar boat a skiff soon thereafter, but this single occasion of use does not change the conventional meaning. Recent exposure does, however, make the word and labeled instances more accessible in memory. Therefore, to the extent that recent exposure affects usage of a noun, usage is being influenced by memory, not by conventional meaning alone. This can happen for either of two reasons. Memory might have an influence because conventional meaning offers a choice; an object might be appropriately labeled in more than one way (something could be called a needle or a syringe). Alternatively, an individual speaker might not be sure of the appropriate label. In the absence of definite knowledge of a word's conventional meaning, one might seek other bases for choice. One basis is to choose names that are consistent with accessible instances.

A likely mechanism for an effect of exposure to instances on naming would be that people use to-be-named (target) objects as retrieval cues for other objects and choose a name in accordance with the retrieved objects. All modern theories of memory agree that events that are recent are easier to retrieve than less recent ones. Events are also easier to retrieve the more similar they are to cues in the retrieval environment (Tulving \& Thomson, 1973). Events that are both recent and similar should be especially available and influential in choosing a name. Therefore, the objects that would most likely be retrieved when cued with a target object would be those that are most similar to the target and that have been recently exposed. In short, conventional meaning is not always accessible or sufficiently constraining when naming. In such cases, we propose that people make use of what they can remem- ber about the name category, more than likely the most similar object or objects encountered in the recent past. We call this the exemplar naming proposal.

The exemplar naming proposal leads to the general hypothesis that naming a target object will be influenced by recent prior exposure to related, labeled examples. The fact that retrieval is proportional to similarity and recency leads, in particular, to the hypothesis that, when the previously exposed objects have different names, the probability of choosing one of those names for the target, relative to the others, should be proportional to the relative similarity between the target and the exposed exemplars. These hypotheses lead to three specific predictions.

First, if one instance from each of two contrasting name categories is presented and named, the one more similar to the target should influence name choice more than the less similar one. Because the exemplar naming hypothesis gives typicality no special status, this effect should occur even if the less similar instance is more typical of its name category than the more similar instance is of its own. In contrast, a standard fixed prototype model states that each familiar name category is associated with a prototype - an ideal or actual instance that is most representative of it - and that objects are named according to the prototype they are most similar to (Homa \& Chambliss, 1975). On this view, any effect of recent exposure to a category instance would be due to its reminding participants of corresponding prototypes. A typical instance would be a better reminder than an atypical one, because it would be more similar to the prototype. Therefore, an effect on target naming should be proportional to the typicality of the exposed instance. Responses to the target should not assimilate to the more similar but less typical instance.

The second prediction is that if two instances of the same name category have been recently exposed, then, because two objects are well within the capacity of memory, both should influence naming. In particular, we assume that the similarity between an object and a comparison set is reduced by adding a less similar element to the set. ${ }^{1}$ When two labeled instances that share a name, one of lower and one of higher similarity, are exposed, they should, therefore, bias naming less toward their name than the higher similarity instance alone. This prediction contrasts with that of a single nearest neighbor model that states that an object is named in accordance with the most similar available instance. Such a model predicts that only the most similar instance affects naming and, therefore, adding a less similar instance to an exposed set should have no effect.

The third prediction draws from the exemplar naming proposal's insensitivity to the typicality of instances and further distinguishes the proposal from a fixed prototype model. Even though a highly typical instance may be a good representative of conventionalmeaning (and it would be, regardless of one's preferred theory of conventional meaning), the presence of a highly typical instance in an exposure set should not overwhelm the influence of other 

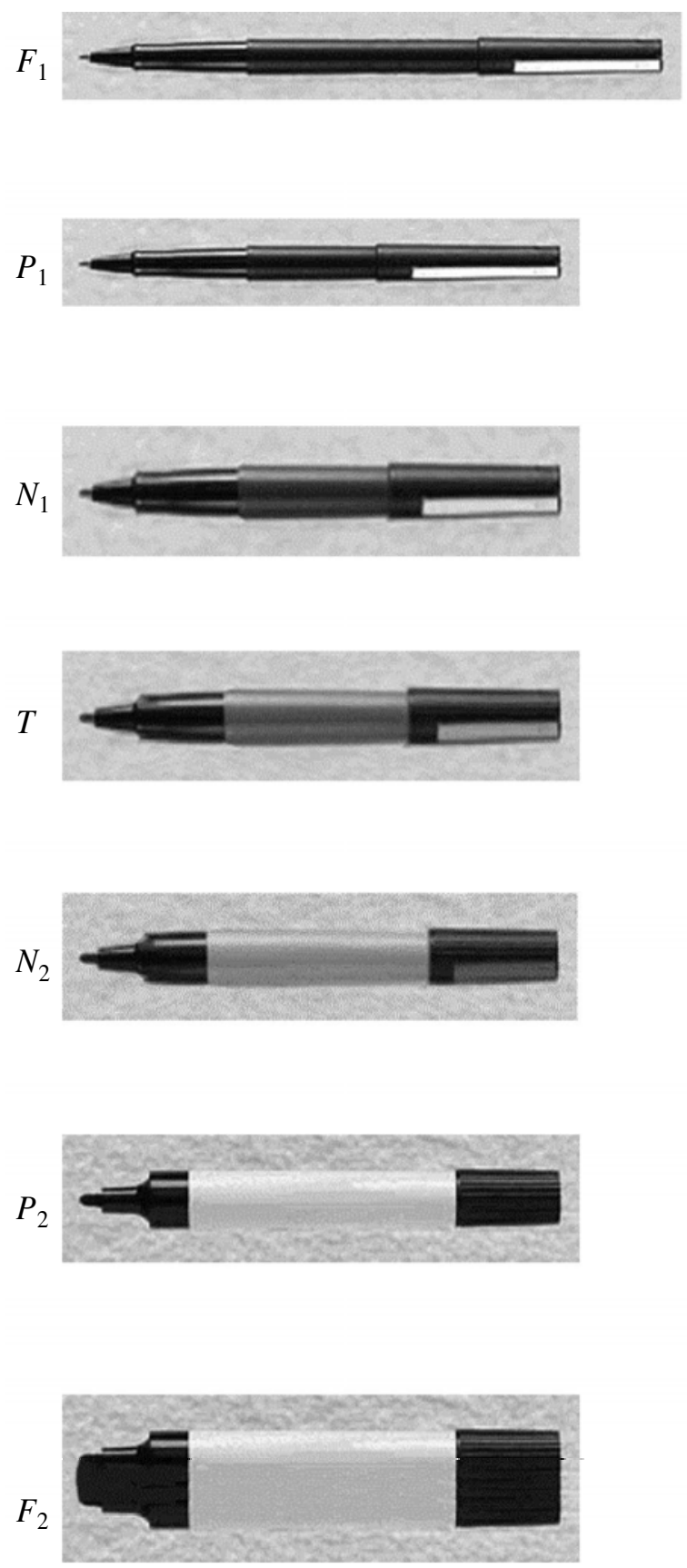

Figure 1. One chain (pen/marker).

instances. All exposed instances, typical or not, should influence name choice. For reasons already described, a fixed prototype model would predict that the influence of typical instances should dominate name choice.

Below, we will report two experiments. Both experiments tested the general hypothesis that recent exposure to related instances affects artifact naming, and both tested the first two specific predictions: (1) Exposure to a similar instance (a neighbor) from one name category and a less similar but typical instance from a second category will bias the naming of an ambiguous target object in the direction of the neighbor, and (2) exposure to a typical instance from the first category along with the neighbor will reduce the bias. The second experiment, in addition, tests the third prediction: (3) All exposed instances will have some influence on name choice.

\section{EXPERIMENT 1}

We constructed chains of photographs of common objects that linked instances of one category to instances of a second category, illustrated for the pen/marker pair in Figure 1. Objects from the middle of the chain could potentially be called by either name. Participants were shown either two or three objects from the chain, depending on the condition. The target object, $T$, is a morph, lying between two typical instances (a typical pen, $P_{1}$, and a typical marker, $P_{2}$ ). In the first condition, the participants were exposed to the typical instance of one category, such as $P_{1}$, and were asked to name it and were also exposed to an atypical instance of the second category that was similar to $T$, in this case $N_{2}$, and were asked to name it. Then we asked people to name $T$ (see Figure 2). We predicted that people would be more likely to call $T$ by Name 2 (marker) than by Name 1 (pen). In a second condition, we added $P_{2}$ to the exposure set, $P_{1}$ and $N_{2}$, and the participants named it. Again, they then named $T$. Because $T$ is, by construction, about as similar to $P_{2}$ as it is to $P_{1}$ and, therefore, less similar, on average, to the Name 2 instances $\left(P_{2}\right.$ and $\left.N_{2}\right)$ than to the Name 2 instance in condition one ( $N_{2}$ alone), we expected less use of Name 2 than in the first condition.

\section{Method}

Materials. The stimulus set consisted of 30 pictures of artifacts created from digital photographs and manipulated using morph and image manipulation software. Twelve of the images were of prototypical objects, two from each of six pairs of artifact categories (chair/stool, plate/bowl, bottle/jar, test tube/graduated cylinder, beaker/flask, and pen/marker). The remaining 18 images were additional exemplars of the categories. The exemplars were created so that they appeared as a graded series of variations between a pair of prototypical objects (illustrated by the set $\left\{P_{1}, N_{1}, T, N_{2}, P_{2}\right\}$ in Figure 1 for the pen/marker series). Each pair of prototypical objects and their three exemplars thus formed a chain of objects from two categories with a nondistinct boundary between them.

The typical instances $\left(P_{1}\right.$ and $\left.P_{2}\right)$ were selected by the experimenters. The validity of the choice was tested using typicality ratings, reported below. The target exemplar $(T)$ was constructed to be roughly equal in similarity to $P_{1}$ and $P_{2}$ via linear interpolation. The two neighbor exemplars $\left(N_{1}\right.$ and $\left.N_{2}\right)$ were roughly equal in similarity to $T$ and their respective typical instances, as we will also validate below.

Design and Procedure. The study had two conditions, varied between participants: The neighbor condition made the typical instance of one category and the neighbor from the other category highly accessible in memory before the naming of the target object by having the participants name them. The prototype + neighbor 
What is this?

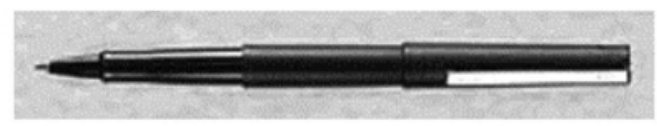

$\left(P_{1}\right)$
What is this?

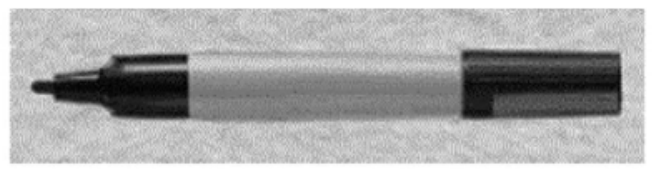

$\left(N_{2}\right)$

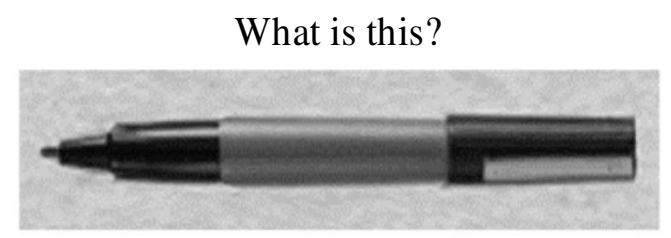

$(T)$

Figure 2. Illustration of the neighbor condition.

condition made both typical instances, as well as one of the neighbors, highly accessible by asking the participants to name all three.

Both conditions had three phases: a naming task, a similarity rating task, and then a typicality rating task, described below. In each phase, the order of items was counterbalanced so that each name category appeared first an equal number of times and each chain (and each item within a chain) appeared the same number of times in each serial position.

Naming. For each chain, the objects in the exposure set were presented one by one on the computer screen (two objects in the neighbor and three in the prototype + neighbor conditions). For each object, the participant was asked "What is this?" and typed his or her response into a response box on the screen. The $T$ object from the same chain was then presented, and the participant was asked to type that object's name into a box. The procedure is illustrated in Figure 2 for the neighbor condition. The two categories within each chain were counterbalanced so that each one supplied the neighbor half the time in each condition.

Similarity. Similarity ratings were collected between $T$ and each of the other four exemplars for each chain. Each pair of pictures was shown side by side, and the participants were asked to rate the similarity of the objects on a scale of 1 (not similar) to 7 (very similar) by typing a number into a reply box on the screen.

Typicality. Typicality ratings were collected for each exemplar of each chain except $T$. The participant was presented with an object and was asked to rate how typical it was of its category on a scale of 1 (not typical) to 7 (very typical) by typing a number into the reply box. The category name was given in the question appearing below the object-for instance,"How typical is this of a pen?"

Participants. Thirty-two Brown University students participated, 16 in each condition, and were paid for their time.

\section{Results}

Table 1 shows the mean proportion of names consistent with the second category. Both predictions were strongly supported. In the neighbor condition, the target was named in line with the category of the neighbor more often than with the category of the typical instance (77\% of the time). This is significantly greater than chance $(50 \%)$ both by participants $[t(15)=6.44, S E=4.2, p<.001]$ and by items $[t(5)=8.81, S E=3.2, p<.001]$. Furthermore, naming was consistent with the neighbor more often in the neighbor condition than in the prototype + neighbor condition (56\%), a difference significant both by participants $[t(15)=4.10, S E=5.1, p<.001]$ and by items $[t(5)=5.06, S E=3.9, p<.01]$. Indeed, the likelihood of picking the category with the exposed neighbor was no greater than chance in the prototype + neighbor condition $[t(15)=1.07, S E=5.8$, n.s., and $t(5)=1.28, S E=6.7$, n.s., by participants and by items, respectively].

The naming proportions include cases in which the participants did not name the nontargets (the previously exposed set) according to the experimenters' expectations. For example, in several cases, $N_{2}$ of Figure 2 was named pen rather than marker. Excluding such cases from analysis ( $28 \%$ of the trials) increased the likelihoods of neighborconsistent naming to $86 \%$ in the neighbor condition and to $69 \%$ in the prototype + neighbor condition. The difference between conditions remained $[t(15)=2.63, S E=$ 6.2 , and $t(5)=2.86, S E=6.7$, both $p \mathrm{~s}<.05$, by participants and by items, respectively]. However, the influence of the neighbor was now evident in both conditions. But this enhanced effect of the neighbor probably reflected an item selection bias. Because more people gave unexpected names to neighbors than to typical instances, these percentages excluded more individuals who tended not to use the neighbors' names than those who tended not to use the typical instances' names. Thus, the measure was biased in favor of the neighbors' names.

Table 1

Mean Proportion of Target Names Consistent with Second Category

Prototype

Neighbor + Neighbor Neighbor + Far Prototype + Far \begin{tabular}{ccccc} 
Experiment & $\left(P_{1}, N_{2}\right)$ & $\left(P_{1}, P_{2}, N_{2}\right)$ & $\left(P_{1}, N_{2}, F_{2}\right)$ & $\left(P_{1}, P_{2}, F_{2}\right)$ \\
\hline 1 & .77 & .56 &
\end{tabular} $2 \quad .72 \quad .61$ .61 .43

Note-The exposure set is shown in parentheses. 
To test the validity of our typicality assignments, we checked that the typicality ratings for the typical instances were high and were higher than the neighbors' ratings. The typical instances' mean was 6.30 on a 7-point scale. This was significantly greater than the neighbor ratings (mean of 5.35) across both participants and items $[t(31)=$ $8.45, S E=0.11, p<.0001$, and $t(11)=5.05, S E=0.19$, $p<.001$, respectively].

We also verified that the similarity relations assumed by our chain construction was correct. Indeed, neighbors were judged to be significantly more similar to the targets than the typical instances were $(6.20$ vs. 4.92$)$ both by participants and by items $[t(31)=14.91, S E=0.086$, by participants; $t(11)=15.05, S E=0.085$, by items; both $p s<.0001]$.

\section{Discussion}

The data supported the general hypothesis that recent exposure to exemplars affects choice of name for an object. They also supported the more specific predictions that people were more likely to name an ambiguous object in a manner consistent with a recently encountered neighbor from one category than they were to name it consistently with a recently encountered typical but less similar instance from a different category and that this effect was reduced - in fact, negligible - when typical instances from both categories were recently encountered.

These data rule out two prominent accounts of categorization as applied to naming. First, they rule out a fixed prototype model (Homa \& Chambliss, 1975). Such a model cannot explain why people named in accordance with the neighbor in the neighbor condition. The data also rule out a pure nearest neighbor model. Such a model cannot explain why presenting a typical instance along with a neighbor in the prototype + neighbor condition reduced the tendency to name targets consistently with their neighbors, relative to the neighbor condition.

Note also that a theory in which people expect categories to occur with equal frequency and adjust their responses accordingly (e.g., Parducci's, 1965, rangefrequency model) cannot explain the data. This possibility could explain why neighbor-consistent naming is less likely in the prototype + neighbor condition, in which two objects have already received the name of the neighbor's category. However, it fails to explain the preference for neighbor-consistent naming in the neighbor condition, in which only one exemplar of each category has been exposed.

Two interpretations of Experiment 1 remain open. Our preferred interpretation, based on the exemplar naming proposal, states that people combined the similarity between the target and all the presented instances of each name category and chose a name in accordance with the most similar exemplar set. If this were the case, the likelihood of naming in accordance with the neighbor should have been greater than .5 even in the prototype + neighbor condition. Perhaps the failure to achieve significance in that condition was simply due to lack of power. A second interpretation rescues prototype theory by adding a new assumption: that the participants used the exposed exemplars to reconstruct a prototype for each category on the spot. According to this possibility, the participants did not spontaneously recall prototypes from long-term memory, but they could recognize the typicality of the presented objects. To name a new object, they constructed a prototype by appealing to the most typical objects they saw. In the neighbor condition, the near neighbor $\left(N_{2}\right)$ was the only object from one category available in working memory and so served as the prototype for that category, whereas the typical instance from the other category $\left(P_{1}\right)$ served as that category's prototype. In the prototype + neighbor condition, the presented typical instances served because they were the most typical objects in working memory for both categories (the only object in the case of $P_{1}$ ). We will refer to this idea as the constructed prototype model. It shares with the fixed prototype model the idea that naming is governed by similarity to a prototype. It differs from the exemplar naming hypothesis in predicting that participants will use only the prototype when it is available in working memory.

\section{EXPERIMENT 2}

Experiment 2 was designed to contrast the exemplar naming and the constructed prototype interpretations of Experiment 1. The exemplar naming proposal states that the influence of individual exemplars should be proportional to their similarity to the target but independent of their typicality. In contrast, a constructed prototype model suggests that the reduction of the neighbor's influence in Experiment 1 in the prototype + neighbor condition, relative to the neighbor-only condition, was due specifically to the presence of the typical instance and that presenting a less typical instance along with the neighbor would have had less influence on neighbor-consistent naming. To evaluate these contrasting predictions, Experiment 2 included a neighbor + far condition. The neighbor from one name category was shown along with a far exemplar, one that was less similar to the target than even the typical instance $\left(F_{1}\right.$ and $F_{2}$ in Figure 1$)$. As before, just the typical instance was shown from the other name category. More generally, this condition should reveal whether, in the presence of a near neighbor, objects other than highly typical instances influence the names given to new objects. If the probability of naming in accordance with the neighbor in this condition is lower than that in the neighbor condition, we can conclude that objects other than near neighbors and typical instances are relevant.

A second new condition also contrasted the exemplar naming and the constructed prototype possibilities by further examining the relevance of other objects in the presence of a typical instance. In this case, we contrasted the typical instance of one category (as usual) with the far exemplar of the other category along with its typical instance (the prototype + far condition). If other objects are ignored in the presence of the typical instance, perfor- 
mance in this condition should equal that in the prototype + neighbor condition. If the target's similarity to all exposed members of a category affects naming, the probability of naming in accordance with the new pair should be less than $50 \%$, because the far item is less similar to the target than is either typical instance.

To examine the generality of prior exposure effects and extend the phenomena, Experiment 2 used a variant of the procedure in Experiment 1. In Experiment 1, the exposure set was always named by the participants themselves. This produced some noise, because the desired names were not always generated. Also, a logical (if not likely) possibility is that the neighbor effect could have depended on this naming procedure. If the experimenter had produced the names, they might have seemed more conventional and appropriate, and therefore, the participants might have relied more on remembered prototypes and less on presented neighbors. To address both of these issues, in Experiment 2 , the exposure set was labeled for the participants.

\section{Method}

The method was identical to that in Experiment 1 in all respects except the following.

Materials. The stimulus set consisted of 56 pictures of artifacts, eight chains (the six from Experiment 1 plus boot/shoe and mop/ broom) consisting of 7 pictures each. The 7 pictures were composed by generating 5 pictures in the same way as in Experiment 1 and concatenating to either end of each chain a far exemplar $\left(F_{1}\right.$ and $\left.F_{2}\right)$, each one similar to a prototypical object, but less similar than its corresponding $N$ and $P$ objects to $T$ (illustrated in Figure 1).

Design and Procedure. The study had four conditions, varied between participants: the two conditions of Experiment 1 plus the neighbor + far and the prototype + far conditions (described above). Unlike Experiment 1, the order of the naming, similarity, and typicality rating tasks was also varied to ensure that we obtained an uncontaminated measure of naming and similarity, in case performance would be affected by the order of tasks. Half of the participants did the naming task first, followed by the similarity rating task and then the typicality rating task. Half were given the similarity task first, followed by typicality and then naming. Each participant was tested on two chains in each condition, so that each chain was presented once per participant.

The procedure differed from Experiment 1 only in that objects other than $T$ were shown in conjunction with a label-that is, the participants only named $T$. The labels were shown by means of a sentence appearing under the object that stated, for example, "this is a pen." The participants were asked to study each object before pressing the space bar to move on to the next object.

Participants. Eighty Brown University students participated for course credit or for remuneration: 40 in the naming-then-similarity task order and another 40 in the similarity-then-naming task order.

\section{Results}

The target object was not given either of the expected names on $1 \%$ of trials. These trials were excluded from analysis. The naming probabilities were first subjected to an analysis of variance, with condition as a within-subjects factor and order of tasks as a between-subjectsfactor. The effect of condition was highly significant $[F(3,234)=$ $8.65, M S_{\mathrm{e}}=0.132, p<.0001$ by participants and $F(3,55)=$
$6.42, M S_{\mathrm{e}}=0.032, p<.001$ by items]. Order had no main effect on how people named, nor did it interact with condition $(F<1$ both by participants and by items in both cases). Table 1 shows the mean probabilities of choosing to name $T$ in accordance with the second category (the one for which something other than just the typical instance was presented) for the various conditions, averaged over the task orders. The results for the first two conditions show that the main results of Experiment 1 were replicated. In the neighbor condition, the target was named in line with the neighbor's category more often than with that of the typical instance ( $72 \%$ of the time). This is significantly greater than chance $(50 \%)$ both by participants $[t(79)=$ 5.82, $S E=3.8, p<.0001]^{2}$ and by items $[t(7)=9.04$, $S E=2.4, p<.0001]$. As in Experiment 1 , naming was consistent with the neighbor more often in the neighbor condition than in the prototype + neighbor condition ( $72 \%$ vs. $61 \%$ ), a significant difference according to a linear contrast by participants [ $t(79)=1.85, S E=5.7, p<.05$ ], but not by items $[t(7)=1.37, S E=9.0, p=.11]$. In this case, the likelihood of picking the category of the presented neighbor was greater than chance in the prototype + neighbor condition across participants $[t(79)=2.64$, $S E=4.3, p=.01]$, but not across items $[t(7)=1.72, S E=$ $5.7, p=.13]$.

The neighbor + far condition was introduced to examine the exemplar naming hypothesis's prediction that instances that are atypical influence naming even if they are not near neighbors of the target. They do: Given exposure to a near neighbor, neighbor-consistent naming was greater in the absence of a far exemplar than in its presence. In fact, the percentage for naming in accordance with the near neighbor's label in the neighbor + far condition was identical to that in the prototype + neighbor condition $(61 \%)$.

This pattern of data cannot be explained by the constructed prototype hypothesis, which would predict that naming should be at chance in the prototype + neighbor condition. It would also predict that naming should be near chance in the neighbor + far condition (if the prototype is constructed by averaging $N_{2}$ and $F_{2}$ ). It could explain the obtained data by assuming that half the participants constructed a prototype by averaging $\mathrm{N}_{2}$ and $\mathrm{F}_{2}$ and half used only $N_{2}$. But such an explanation would be ad hoc and unmotivated.

The prototype + far condition was introduced to further test the constructed prototype hypothesis by examining its prediction that the presence of the typical instance would cause the participants to ignore other instances. Again, the exemplar naming hypothesis predicts that all exemplars should exert some influence. The fact that performance was greater than chance in the prototype + neighbor condition already suggests that the typical instance did not overwhelm the field in the sense claimed by the prototype hypothesis. The prototype + far condition casts further doubt on this hypothesis, because the naming probability was .43 , less than .5 , suggesting that some attention 
Table 2

Mean Typicality Ratings for Each Type of Item

\begin{tabular}{cccc}
\hline Experiment & Neighbors & Prototypes & Far Exemplars \\
\hline 1 & 5.35 & 6.30 & \\
2 & 5.43 & 6.08 & 4.93 \\
\hline
\end{tabular}

may have been paid to the far exemplar. However, the difference was only marginally significant across participants $[t(79)=1.74, S E=4.0, p=.09]$ and was not significant across items $[t(7)=1.21, S E=4.7$, n.s.].

We again tested the validity of our typicality assignments. Mean typicality ratings for each item type are shown in Table 2. Again, ratings for the typical instances were high and were higher than the neighbors' ratings. The typical instance means were 6.08 on a 7-point scale. They were significantly greater than the neighbor ratings (mean of 5.43) across both participants and items $[t(79)=$ 14.14, $S E=0.046, p<.0001$, and $t(15)=3.97, S E=$ $0.16, p<.01$, respectively]. As was expected, the typical instance ratings were also significantly higher than the far exemplar ratings [mean of $4.93 ; t(79)=17.12, S E=$ $0.068, p<.0001$ by participants and $t(15)=6.32, S E=$ 0.18 by items; all $p \mathrm{~s}<.0001]$.

We again verified the similarity relations assumed by our chain construction. Mean similarity ratings for each nontarget item type are shown in Table 3. Neighbors were judged to be significantly more similar to the targets than the typical instances were both by participants and by items $[t(79)=26.12, S E=0.056$, and $t(15)=14.29, S E=$ 0.10 , respectively]. Also, far exemplars were judged to be significantly less similar than typical instances $[t(79)=$ $18.81, S E=0.045$ by participants and $t(15)=6.13, S E=$ $0.15, p<.0001$ by items].

In contrast to Livingston et al. (2001), no evidence of an effect of naming on similarity judgments was obtained. For each nontarget, we compared the rating of similarity to the target given by the group that rated similarity after the naming task with the rating of similarity given by the group that rated similarity before naming. No systematic differences were found.

\section{Discussion}

The main effects of Experiment 1 were replicated. Name choices were more consistent with a recently exposed neighbor than with a recently exposed typical instance, and the effect was reduced by also exposing a typical instance from the neighbor's category. These effects generalized the results of Experiment 1, inasmuch as they were obtained - unlike in Experiment 1-when the nontarget pictures were labeled for the participants, thus emphasizing the conventionality of the names.

The neighbor + far and the prototype + far conditions of Experiment 2 were intended to shed light on the participants' thought processes in the prototype + neighbor condition. The finding that performances in the prototype + neighbor and the neighbor + far conditions were identical could, taken by itself, imply that exposure to instances other than neighbors does not affect naming. But this conclusion is not consistent with the difference observed between the prototype + neighbor and the neighbor conditions. Adding a typical instance to a neighbor in the exposed set reduced the effect of neighbor-consistent naming, and so the typical instance must have been attended to. Therefore, the general trend of the data was consistent with the exemplar naming proposal that all the objects in exposed sets influence naming judgments and that their influence is proportional to their similarity to the target.

This conclusion was further supported by the prototype + far condition, the only condition showing a minority of names consistent with the category corresponding to the neighbor category in all the other conditions. A bias against using that name could be attributed only to exposure to the far exemplar. The bias did not reach statistical significance in this experiment, perhaps because the far exemplar had only a weak effect and the more similar instance- the typical one-was given more weight in the naming judgment.

One possible result of this experiment was that the exposure of a far instance would increase the perceived scope of the name, so that naming proportions in the neighbor + far and the prototype + far conditions would have been greater than those in the neighbor and the prototype + neighbor conditions, respectively. That this did not happen implies that the effect of exposure is not merely to cue the range of application of a label.

Furthermore, the prototype + far condition reinforced the conclusion that the effects we report cannot be attributed to participants' trying to construct categories of equal size and, more generally, cannot be attributed to the number of objects from each name category that are exposed. Exposing people to one instance from one category versus two instances from a different category can lead to a preference for the two-instance category (prototype + neighbor and neighbor + far conditions) or to no preference (prototype + far condition).

Finally, note that none of the effects can be attributed to changes in accessibility of labels, as opposed to labeled instances. In every condition of both experiments, labels for both categories were exposed (either generated or presented) prior to target naming.

\section{GENERAL DISCUSSION}

\section{Summary and Conclusion}

These experiments have shown that prior exposure to familiar objects and their names affects how an ambiguous target object is named, and they shed some light on

Table 3

Mean Similarity Ratings to Targets for Each Type of Item

\begin{tabular}{cccc}
\hline Experiment & Neighbors & Prototypes & Far Exemplars \\
\hline 1 & 6.20 & 4.92 & \\
2 & 6.10 & 4.63 & 3.73 \\
\hline
\end{tabular}


how this occurs. In every condition, we pitted exposure to a typical instance with one name against exposure to one or two instances from a contrasting name category. Both experiments showed that when the contrast set consisted of a neighbor-a single instance more similar to the target than the typical instance-people tended to name the target with the name of the contrasting category (the neighbor condition). This effect was reduced, but not completely eliminated, when a typical instance of the contrasting category was also exposed (the prototype + neighbor condition). These results suggest that naming is biased in the direction of recently exposed instances in proportion to their similarity to the target.

Experiment 2 showed that when a typical instance from one category was pitted against the neighbor, as well as against an atypical and dissimilar exemplar from the contrast category (neighbor + far condition), people were just as likely to use the contrast category label for the target as they were in the prototype + neighbor condition. When the exposed contrast category instances were the typical and a dissimilar instance (prototype + far condition), the likelihood of using the contrast category name for the target was substantially reduced. The effect of exposure was proportional to the similarity between the target and all the presented instances, whether they were typical or not.

We conclude that ambiguous objects are not named only in relation to either fixed prototypes or single nearest neighbors. Rather, all recently encountered objects affect name choice, in proportion to their similarity to the target. This conclusion is consistent with a process model of naming that posits that people use to-be-named objects as retrieval cues for other objects and name them in accordance with the retrieved objects. On this view, exposure affects naming because recency aids retrieval, and naming is proportional to similarity because retrieval is proportional to similarity.

The data are also consistent with a variant of this account: The dominant strategy might have been to name with respect to a near neighbor but to adjust naming judgments in a direction consistent with other instances that came to mind. That adjustment might have been independent of the other instances - that is, of relatively constant magnitude no matter which other objects were exposed.

\section{Implications}

We have shown a substantial effect of prior exposure on naming. The implication of this finding is that the naming of ambiguous objects is guided by specific personal experiences. This, in turn, implies that naming is not only flexible, but also idiosyncratic. Clearly, naming must be constrained by a word's conventional meaning; otherwise, people would not be able to communicate. But these data show that other constraints can also matter. People will use whatever is most accessible in memory when choosing a name.

Our conclusion provides a reconciliation of sorts for the prototype versus exemplar model debate. Our view is that each of these hypotheses captures one constraint on ar- tifact naming. The prototype hypothesis captures the goal of generating names that are consistent with conventional meaning. The exemplar hypothesis captures the uncertainty that knowledge of conventional meaning can exhibit. When uncertain about the boundary conditions of a label, reliance on specific accessible exemplars whose names have been established is a good strategy for choosing a name that will be effective for communication.

Unlike most examinations of the role of typicality in categorization, the experiments we have reported used familiar, well-entrenched categories, such as shoes, boots, mops, and brooms. Almost all previous research has used experimenter-constructed stimuli placed into experimenter-determined categories for which category knowledge is limited to the context of the experiment (for exceptions, see Brooks et al., 1991; Heit \& Barsalou, 1996; Storms, De Boeck, \& Ruts, 2000). Such artificial categories enable control over a number of variables, but they also reduce participants' ability to use normal naming strategies. For example, instead of asking themselves what the language community's understanding of an object is and how it relates to other objects, the participants must ask themselves what kind of response is deemed correct in this particular experiment. An unintended consequence of such a change is that prototypical instances may have less influence than they would have with more familiar categories. When stimuli are made up, participants might focus on the experimentally determined category boundary, especially if they are aware that many of the test items lie on or near it, rather than on the "meaning" of the category label, as represented by the prototype.

These results may or may not generalize to other types of categories. The crucial question is the degree to which naming an object elicits an appeal to memory for the names of related objects. Other sorts of objects may elicit other sorts of naming strategies. Presumably, many cases of legal, scientific, and bureaucratic classification involve choosing a name according to some prescribed procedure (one official designation of some residents of the United States is "legal alien," a label that rarely derives from appeals to memory for a related instance). Perhaps more complex artifacts than ours or some natural kinds would show less of an effect of specific prior experiences, because naming them might require more analysis of the properties associated with conventional meaning than would be required for simple artifacts. We doubt it, though. We suspect that there is less variability and more confidence in the conventional meanings associated with simple artifacts than in those associated with most other types of objects. Although the terms people use to refer to simple artifacts may have complex extensions (Malt et al., 1999), we suspect that people have more firmly held beliefs about category prototypes and greater familiarity with representative instances than they do for most other kinds of terms. As a result, we would expect more appeal to contextually driven influences and to prior occasions of use - and therefore, exemplar influences - when other 
kinds of objects are named. In this sense, we believe that the domain we have chosen provides a strong test of the role of exemplar-specific memory.

If a particular effect of memory on naming is persistent and broad, not only may it contribute to how a word is used on particular occasions, but also, over a long period of time, it may feed back and affect the word's conventional meaning. To illustrate, as militaries have become more sophisticated, use of the word weapon has been stretched to cover a set of things that could not even be imagined 100 years ago. As the use is extended in some directions (and perhaps contracted in others, when obsolete weapons become unfamiliar to a language community), the examples of weapon retrieved when using the word inevitably change. The prototype associated with the word will ultimately shift to reflect the set of examples most often retrieved. Shorter term influences of memory on naming thus cumulate to have longer term influences on conventional meaning. Memory is part of an iterative, dynamic process of evolution of word meaning.

\section{REFERENCES}

Bless, H., Igou, E. R., Schwarz, N., \& WÄNKe, M. (2000). Reducing context effects by adding context information: The direction and size of context effects in political judgment. Personality \& Social Psychology Bulletin, 26, 1036-1045.

BLoom, P. (1996). Intention, history, and artifact concepts. Cognition, 60, 1-29.

Brennan, S., \& Clark, H. H. (1996). Conceptual pacts and lexical choice in conversation. Journal of Experimental Psychology: Learning, Memory, \& Cognition, 22, 1482-1493.

Brooks, L. R., Norman, G. R. \& Allen, S. W. (1991). Role of specific similarity in a medical diagnostic task. Journal of Experimental Psychology: General, 120, 278-287.

Brown, R. W. (1958). How shall a thing be called? Psychological Review, 65, 14-21.

Gennari, S. P., Sloman, S. A., Malt, B. C., \& Fitch, W. T. (2002). Motion events in language and cognition. Cognition, 83, 49-79.

Grice, H. P. (1975). Logic and conversation. In P. Cole \& J. Morgan (Eds.), Syntax and semantics: Vol. 3. Speech acts (pp. 41-58). New York: Academic Press.

HeIt, E. (1992). Categorization using chains of examples. Cognitive Psychology, 24, 341-380.

HeIT, E. \& BARSAlou, L. W. (1996). The instantiation principle in natural language categories. Memory, 4, 413-451.

Hintzman, D. L. (1986). Schema abstraction in a multiple-trace memory model. Psychological Review, 93, 411-428.

Homa, D., \& Chambliss, D. (1975). The relative contribution of common and distinctive information on the abstraction from ill-defined categories. Journal of Experimental Psychology: Human Learning \& Memory, 1, 351-359.

LAKOFF, G. (1987). Women, fire, and dangerousthings: What categories reveal about the mind. Chicago: University of Chicago Press.

Livingston, K. R., Andrews, J. K., \& Dwyer, P. (2001). Ties that bind: Reconciling discrepancies between categorization and naming. In Proceedings of the 23rd Annual Conference of the Cognitive Science Society (pp. 558-563). Mahwah, NJ: Erlbaum.

Malt, B. C., Sloman, S. A., Gennari, S., Shi, M., \& Wang, Y. (1999). Knowing versus naming: Similarity and the linguistic categorization of artifacts. Journal of Memory \& Language, 40, 230-262.
Medin, D. L., \& Ross, B. H. (1989). The specific character of abstract thought: Categorization, problem-solving, and induction. In R. Sternberg (Ed.), Advances in the psychology of human intelligence (Vol. 5, pp. 189-223). Hillsdale, NJ: Erlbaum.

Medin, D. L., \& Schaffer, M. M. (1978). Context theory of classification learning. Psychological Review, 85, 207-238.

Millikan, R. G. (1998). A common structure for concepts of individuals, stuffs, and basic kinds: More mama, more milk and more mouse. Behavioral \& Brain Sciences, 22, 55-65.

NosofSKY, R. M. (1988). Similarity, frequency, and category representation. Journal of Experimental Psychology: Learning, Memory, \& Cognition, 14, 54-65.

ParducCi, A. (1965). Category judgments: A range-frequency model. Psychological Review, 72, 407-418.

Putnam, H. (1975). The meaning of "meaning.” In H. Putnam (Ed.), Mind, language, and reality: Philosophical papers (Vol. 2, pp. 131193). Cambridge: Cambridge University Press.

Ross, B. H., Perkins, S. J., \& Tenpenny, P. L. (1990). Remindingbased category learning. Cognitive Psychology, 22, 460-492.

Sloman, S. A., \& AHN, W.-K. (1999). Feature centrality: Naming versus imagining. Memory \& Cognition, 27, 526-537.

Spalding, T. L., \& Ross, B. H. (1994). Comparison-based learning: Effects of comparing instances during category learning. Journal of Experimental Psychology: Learning, Memory, \& Cognition, 20, 12511263.

Spalding, T. L., \& Ross, B. H. (2000). Concept learning and feature interpretation. Memory \& Cognition, 28, 439-451.

Storms, G., De Boeck, P., \& Ruts, W. (2000). Prototype and exemplarbased information in natural language categories. Journal of Memory \& Language, 42, 51-73.

TAY LOR, J. R. (1995). Linguistic categorization: Prototypes in linguistic theory (2nd ed.). Oxford: Oxford University Press.

Tulving, E. \& Thomson, D. M. (1973). Encoding specificity and retrieval processes in episodic memory. Psychological Review, 80, 352373.

Tversky, A. (1977). Features of similarity. Psychological Review, 84, 327-352.

WARD, L. M., \& LOCKHEAD, G. R. (1970). Sequential effects and memory in category judgments. Journal of Experimental Psychology, 84, 27-34.

Wedell, D. H., \& Pettibone, J. C. (1999). Preference and the contextual basis of ideals in judgment and choice. Journal of Experimental Psychology: General, 128, 346-361.

\section{NOTES}

1. We are assuming that the similarity between an object and a comparison set is reduced by adding a less similar element to the set; in essence, that similarity is computed by averaging the similarity between the object and all the exemplars in the set. This assumption is not shared by several prominent exemplar models of categorization that assume similarities are added rather than averaged (e.g., Hintzman, 1986; Nosofsky, 1988). However, even those models predict that adding a dissimilar item can reduce similarity if similarities are negative (as allowed by, e.g., Tversky's, 1977, similarity scale). Note that the models otherwise make the counterintuitive prediction that an object may be seen as more likely to belong to a category that contains a large number of dissimilar objects than to a category that contains a small number of relatively similar objects.

2 . This and the following inferential tests across participants violate the assumption of normality. Because each participant gave two judgments in each condition, their individual scores are trinary $(0, .5$, or 1$)$.

(Manuscript received May 17, 2001; revision accepted for publication April 20, 2002.) 